\title{
Basis of cerulenin resistance of two strains of Candida albicans
}

\author{
Gail McElhaney-Feser and Ronald L. Cihlar \\ Author for correspondence: Ronald Cihlar. Tel: +1 202687 1136. Fax: +1 2026871800.
}

Department of Microbiology and Immunology, Georgetown University, Washington, DC 20007, USA

\begin{abstract}
The basis of cerulenin resistance of Candida albicans strains 4918-2 and 491810 has been investigated. Parasexual genetic analyses established that cerulenin resistance to concentrations of at least $5 \mu \mathrm{g} \mathrm{ml}^{-1}$ is dominant in both strains. The results also showed that strain 4918-2 is heterozygous for resistance, while the change from resistance to sensitivity of strain 4918-10 is reversible. Experiments to define the mechanism(s) responsible for resistance focused on cerulenin uptake and fatty-acid synthase activity. Cerulenin uptake by strains $4918-2$ and $4918-10$ was $24 \%$ of that of the wild-type (strain 4918). Uptake was restored in UV-induced cerulenin-sensitive segregants of strains 4918-2 and $4918-10$, and varied from $63 \%$ to $200 \%$ of parental values. Fattyacid synthase from strains $4918-2$ and 4918-10 was resistant to cerulenin as judged by differences in the inactivation of the enzyme by the agent. However, inactivation kinetics of fatty-acid synthase of cerulenin-sensitive segregants did not revert to the parental inactivation profile. Further investigation showed that nine out of ten segregants were resistant to cerulenin at concentrations between 1 and $4 \mu \mathrm{g} \mathrm{ml}^{-1}$ while strain 4918 was sensitive to cerulenin at all concentrations tested. Thus, the results suggest that alteration of fatty-acid synthase and changes in permeability contribute to total cerulenin resistance of each strain.
\end{abstract}

Keywords: Candida albicans, cerulenin, resistance, fatty-acid synthase, uptake

\section{INTRODUCTION}

Candida albicans is an important opportunistic fungal pathogen which promotes a variety of disorders ranging from localized infection to systemic disease (Odds, 1988). Potential virulence factors include germ tube formation (Cutler, 1991; De Bernardis et al., 1993), protease production (Ross et al., 1990; Cutler, 1991), and phenotypic switching (Soll, 1992). Adherence to host tissues is also critical in the establishment of infection (Hostetter, 1994).

We have previously described avirulent mutant strains of C. albicans (Hoberg et al., 1986). The strains, designated 4918-2 and 4918-10, were isolated as spontaneous cerulenin-resistant derivatives of $C$. albicans, strain 4918 (Cihlar et al., 1984). Both mutant strains were subsequently shown to be relatively avirulent in the rabbit model of endocarditis (Calderone et al., 1986), and strain 4918-10 showed reduced virulence in a murine model of vaginal candidiasis (Lehrer et al., 1986). As both mutant strains showed poor adherence relative to the parental

Abbreviation: MPA, mycophenolic acid. strain (Calderone et al., 1986; Hoberg et al., 1986), it was reasoned that this trait might account for the differences in virulence observed. In this regard, analysis of the cell wall/membrane of the mutant strains identified a variety of differences in comparison to the parental strain, including protein profile (Hoberg et al., 1986), mannan composition and linkage (Saxena et al., 1989, 1990), and reduced amounts of $\mathrm{iC} 3 \mathrm{~b}$ receptor (Ollert et al., 1990). More recently, fatty-acid synthase, the target of cerulenin (Ōmura, 1981), has been purified from the parental and mutant strains (McElhaney-Feser \& Cihlar, 1994). The kinetics of inactivation of the respective fatty-acid synthases in the presence of cerulenin showed that the enzyme from each mutant was more resistant to cerulenin than the parental enzyme, although such alteration alone did not account for the level of in vivo resistance observed.

Thus, despite extensive phenotypic characterization of the mutant strains, the basis of cerulenin resistance has not been established. Likewise, no information concerning putative genetic changes that might be responsible for resistance has been reported. We have performed investigations addressing these issues, and they are discussed herein. 


\section{METHODS}

Strains and culture conditions. C. albicans 4918 (Manning \& Mitchell, 1980) was the wild-type strain used in all experiments. Strains 4918-2 and 4918-10 are spontaneous, cerulenin-resistant derivatives of strain 4918 (Cihlar et al., 1984). C. albicans strain 1033 was a gift from Dr Stuart Scherer (University of Minnesota School of Medicine, Minneapolis, MN, USA) and has been described by Goshorn \& Scherer (1989). Strains were maintained by weekly subculture on MSA agar (Difco) from stocks stored at $-70^{\circ} \mathrm{C}$. Before each experiment appropriate medium was inoculated with a single colony and $18 \mathrm{~h}$ cultures were prepared. These in turn were used to inoculate fresh medium, and cultures were incubated at $27^{\circ} \mathrm{C}$ with rotary shaking to a cell density of approximately $1 \times 10^{7} \mathrm{cells} \mathrm{m}^{-1}$. For genetic experiments strains were grown in Phytone-peptone broth (BBL) containing $0.1 \%$ glucose or YEPD $(2 \%, w / v$, Bacto yeast extract, $1 \%, \mathrm{w} / \mathrm{v}$, Bacto peptone, containing $2 \%, \mathrm{w} / \mathrm{v}$, glucose) medium at $27^{\circ} \mathrm{C}$ in order to obtain yeast cells.

Parasexual genetic experiments. UV-irradiation conditions were as described by Whelan \& Magee (1981), with the exception that cells were plated on modified Sabouraud agar at 150-200 c.f.u. prior to irradiation. Irradiation was performed to yield $85-90 \%$ survival. After incubation for $5 \mathrm{~d}$ at $25^{\circ} \mathrm{C}$ colonies were replica-plated to M63 medium (Miller, 1972) and M63 medium supplemented with $5 \mu \mathrm{g}$ cerulenin $\mathrm{ml}^{-1}$ (Sigma). Plates were scored after $5 \mathrm{~d}$. Replicas of colonies showing sectoring were picked, purified by streaking and retested for cerulenin sensitivity or resistance, depending on the experiment. Spheroplast fusion experiments were performed as reported elsewhere (Goshorn \& Scherer, 1989). Fusion products were selected on the basis of resistance to mycophenolic acid (MPA), and were subsequently tested for resistance to cerulenin.

Cerulenin MIC determinations. In order to determine the resistance level of individual strains, M63 agar containing cerulenin at the appropriate concentrations was first dispensed into 96-well titre dishes. Subsequently, $10^{2}$ c.f.u. of each test strain were inoculated to the surface of each well. Plates were incubated at $24{ }^{\circ} \mathrm{C}$ and scored for growth at 2 and $5 \mathrm{~d}$.

Measurement of fatty-acid synthase activity. Fatty-acid synthase activity was assayed as described by Lynen (1969). Crude enzyme preparations were obtained from yeast cultures of the appropriate strains as previously reported (McElhaneyFeser \& Cihlar, 1994). Reaction mixtures $(1 \mathrm{ml})$ comprised (final concentrations): $0.4 \mathrm{M}$ potassium phosphate buffer, $\mathrm{pH} 7 \cdot 3$, containing $0.125 \mu \mathrm{M}$ dithiothreitol, $50 \mu \mathrm{M}$ acetyl-CoA, $100 \mu \mathrm{M}$ NADPH, enzyme preparation, and, in some assays, appropriate concentrations of cerulenin (Sigma). Following incubation at $37^{\circ} \mathrm{C}$ for 5 min malonyl-CoA containing $0.5 \mu \mathrm{Ci}(18.5 \mathrm{kBq})$ $\left[2{ }^{14} \mathrm{C}\right.$ malonyl-CoA $\quad\left(58.8 \mathrm{mCi} \mathrm{mmol}^{-1}, \quad 2.17 \mathrm{GBq} \mathrm{mmol}^{-1}\right.$; Amersham) was added to a final concentration of $100 \mu \mathrm{M}$. Reactions were terminated after $10 \mathrm{~min}$ by addition of $30 \mu \mathrm{l}$ $60 \%(\mathrm{v} / \mathrm{v})$ perchloric acid and $1 \mathrm{ml}$ absolute ethanol. Fatty acids were extracted with light petroleum $(4 \times)$ (boiling point range $30-75^{\circ} \mathrm{C}$ ), extracts were combined and dried in scintillation vials, and finally solubilized in $3070 \mathrm{~B}$ scintillation fluid (Research Products International). Radioactivity was assayed in a Beckman LSC-230 scintillation counter and enzyme activity was expressed in $U$ (mg protein $)^{-1}(1$ unit = amount of enzyme required to catalyse incorporation of $1 \mathrm{nmol}\left[{ }^{14} \mathrm{C}\right]$ malonate into fatty acid $\min ^{-1}$ under assay conditions).

Measurement of cerulenin uptake. Overnight cultures grown in YEPD were diluted in fresh broth to an $\mathrm{OD}_{600}$ of 0.2 and then incubated at $27^{\circ} \mathrm{C}$ until reaching a density of $1 \times 10^{7}$ cells $\mathrm{ml}^{-1}$. Cells were collected by centrifugation, washed once with YEPD and finally resuspended in fresh media. Subsequently, cerulenin was introduced to cultures at a final concentration of either $5 \mu \mathrm{g} \mathrm{m}^{-1}$ or $10 \mu \mathrm{g} \mathrm{ml}^{-1}$ containing $\left[{ }^{3} \mathrm{H}\right]$ cerulenin at $10 \mu \mathrm{Ci}$

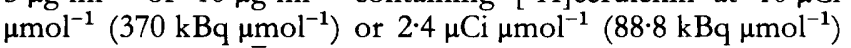
(gift from Dr S. Ōmura, Kitasato Institute, Tokyo, Japan), respectively, and aliquots were removed at appropriate time intervals. Cells were collected by filtration utilizing Whatman GF/C filters, and then washed with $30 \mathrm{ml}$ cold PBS $(0.145 \mathrm{M}$ $\mathrm{NaCl}, 0.15 \mathrm{M}$ sodium phosphate). After drying, filters were placed in 3070B scintillation fluid and subsequently radioactivity was assayed in a Beckman LSC-230 liquid scintillation counter. After correction for non-specific binding of radiolabel, data were expressed as ng $\left[{ }^{3} \mathrm{H}\right]$ cerulenin per $10^{7}$ cells $\mathrm{ml}^{-1}$.

\section{RESULTS}

\section{UV-induced mitotic recombination}

In order to gain insight into whether 4918-2 and/or 491810 were either heterozygous or homozygous for cerulenin resistance, the incidence of recovery of cerulenin-sensitive segregants after UV-irradiation was measured. Data shown in Table 1 summarize the results of these experiments. In the case of 4918-2, sensitive clones were readily isolated after irradiation. Subsequent irradiation of five sensitive clones obtained from three independent experiments failed to yield cerulenin-resistant segregants, indicating that the initial mutation in 4918-2 rendered the strain heterozygous for resistance.

Identical experiments with 4918-10 yielded incongruous results. Following irradiation, sensitive clones were isolated at frequencies similar to that noted for 4918-2 suggesting that the strain was heterozygous for resistance. However, UV-treatment of selected cerulenin-sensitive isolates gave rise to resistant clones at frequencies comparable to those observed for conversion of resistance to sensitivity. The data, therefore, show that the events responsible for the change from sensitivity to resistance and its reciprocal are reversible.

Further perspective concerning cerulenin resistance of strain 4918-10 was obtained from spheroplast fusion experiments. Strain 4918-10 was fused with $C$. albicans strain 1033. The latter strain provides for direct selection of prototrophic variants by virtue of a dominant marker for MPA resistance (Goshorn \& Scherer, 1989). Analysis of 242 fusion products showed $80 \%$ to be ceruleninresistant, supporting the notion that the ceruleninresistant phenotype is dominant in 4918-10. Similar experiments could not be performed to confirm dominance of strain $4918-2$ as the strain proved resistant to MPA.

\section{Fatty-acid synthase activity}

It was previously demonstrated that fatty-acid synthase from both 4918-2 and 4918-10 showed altered inactivation kinetics in the presence of cerulenin as compared to the enzyme from the parental strain (McElhaney-Feser \& Cihlar, 1994). Thus, fatty-acid synthase from ceruleninsensitive clones obtained in mitotic recombination experiments was surveyed to determine if restoration of wildtype enzyme inactivation kinetics accompanied reversion to cerulenin sensitivity. Representative data are depicted in Fig. 1, and demonstrate that inactivation kinetics of 
Table 1. UV-induction and analysis of cerulenin-sensitive segregants of $C$. albicans strains 4918-2 and 4918-10

\begin{tabular}{|cccccc|}
\hline Strain & $\begin{array}{c}\text { Survival } \\
\text { frequency }\end{array}$ & $\begin{array}{c}\text { Frequency of } \\
\text { cerulenin- } \\
\text { sensitive } \\
\text { segregants }\end{array}$ & $\begin{array}{c}\text { No. of } \\
\text { segregants } \\
\text { UV-treated }\end{array}$ & $\begin{array}{c}\text { Survival } \\
\text { frequency }\end{array}$ & $\begin{array}{c}\text { Frequency of } \\
\text { cerulenin- } \\
\text { resistant } \\
\text { segregants }\end{array}$ \\
\hline $4918-2$ & 0.96 & $5.88 \times 10^{-3}$ & 2 & 0.76 & 0 \\
& 0.90 & $6.56 \times 10^{-3}$ & 2 & 0.82 & 0 \\
& & & & 0.73 & 0 \\
& 0.90 & $5.56 \times 10^{-3}$ & 1 & 0.91 & 0 \\
& 0.82 & $8.18 \times 10^{-3}$ & 1 & 0.61 & 0 \\
& 0.74 & $5.93 \times 10^{-3}$ & 2 & 0.62 & $2.50 \times 10^{-3}$ \\
& & & & 0.88 & $5.58 \times 10^{-3}$ \\
& 0.84 & $9.86 \times 10^{-3}$ & 1 & 0.81 & $5.68 \times 10^{-3}$ \\
& 0.92 & $9.33 \times 10^{-3}$ & 1 & 0.79 & $5.05 \times 10^{-3}$ \\
\hline
\end{tabular}
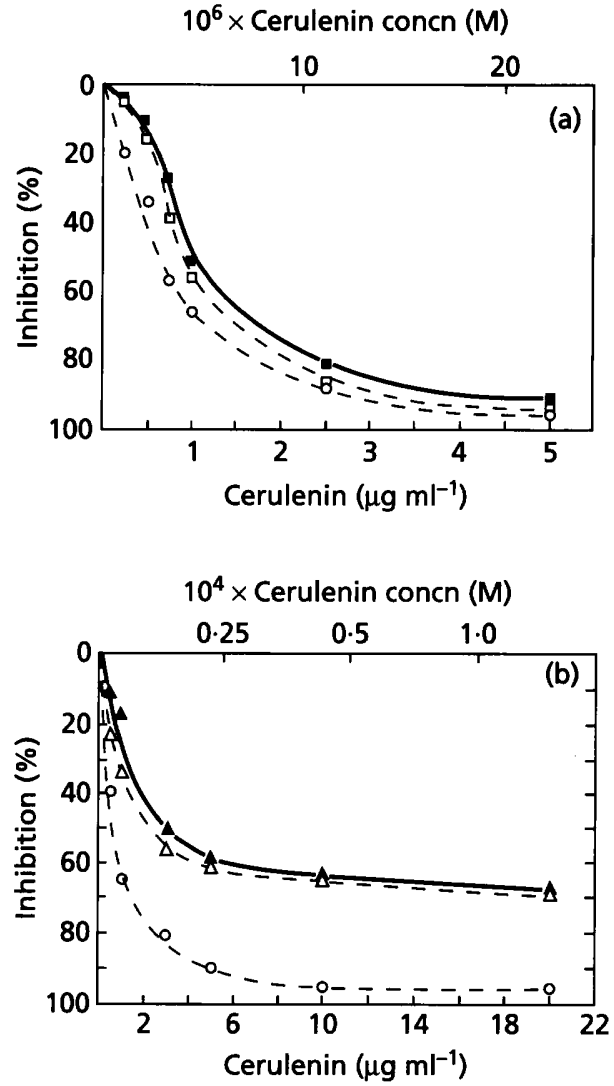

Fig. 1. Effect of cerulenin on fatty-acid synthase activity. Crude enzyme preparations were obtained and assayed as described by McElhaney-Feser \& Cihlar (1994). Data were plotted as percentage inhibition from initial activity as a function of increasing cerulenin concentrations. (a) Fatty-acid synthase activity of strains $4918(O), 4918-2(\square)$ and cerulenin-sensitive segregant $004(\square)$. Results comparable to those for strain 004 were obtained for the other cerulenin-sensitive segregants of strain 4918-2 discussed. (b) Fatty-acid synthase activity of strains $4918(O), 4918-10(\boldsymbol{A})$ and cerulenin-sensitive segregant LR71 $(\triangle)$. Results comparable to those for strain LR71 were obtained for the other cerulenin-sensitive segregants of strain 4918-10 discussed.

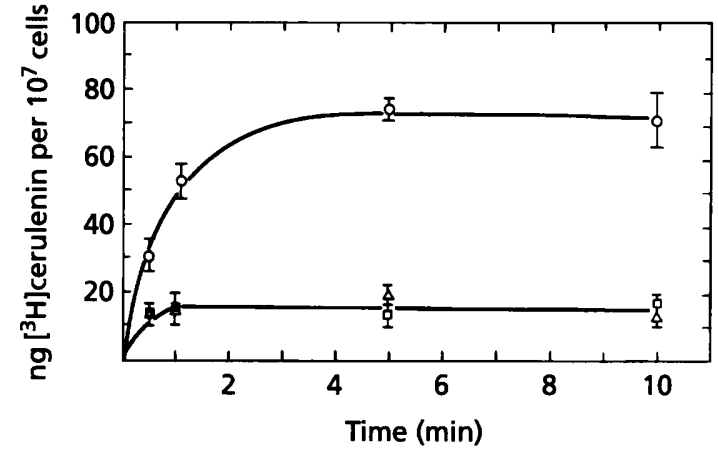

Fig. 2. Cerulenin uptake by strains $4918(O), 4918-2(\square)$ and 4918-10 $(\triangle)$. Uptake was measured as described in Methods. The results presented are from three independent experiments, in which $200 \mu \mathrm{l}$ aliquots were collected after the introduction of $\left[{ }^{3} \mathrm{H}\right]$ cerulenin $\left(10 \mu \mathrm{Ci} \mu \mathrm{mol}^{-1} ; 370 \mathrm{kBq} \mu \mathrm{mol}^{-1}\right)$ at the time points indicated. Uptake was then measured as described in Methods.

fatty-acid synthase of cerulenin-sensitive clones derived from both 4918-2 and 4918-10 did not revert to the wildtype, but rather maintained the profile of the respective mutant strain. It should be noted that results obtained with crude enzyme preparations of strains $4918,4918-2$ and 4918-10 showed no significant differences from results reported earlier using purified enzyme preparations (McElhaney-Feser \& Cihlar, 1994).

\section{Cerulenin uptake}

Since mutation of fatty-acid synthase does not account for the level of in vivo cerulenin resistance of strains 4918-2 and 4918-10 (McElhaney-Feser \& Cihlar, 1994), and since increased cerulenin sensitivity is not accompanied by reestablishment of wild-type inactivation kinetics of fattyacid synthase, other mechanisms must contribute to the resistant phenotype. In this context, the permeability of the strains to cerulenin was investigated. Fig. 2 illustrates 


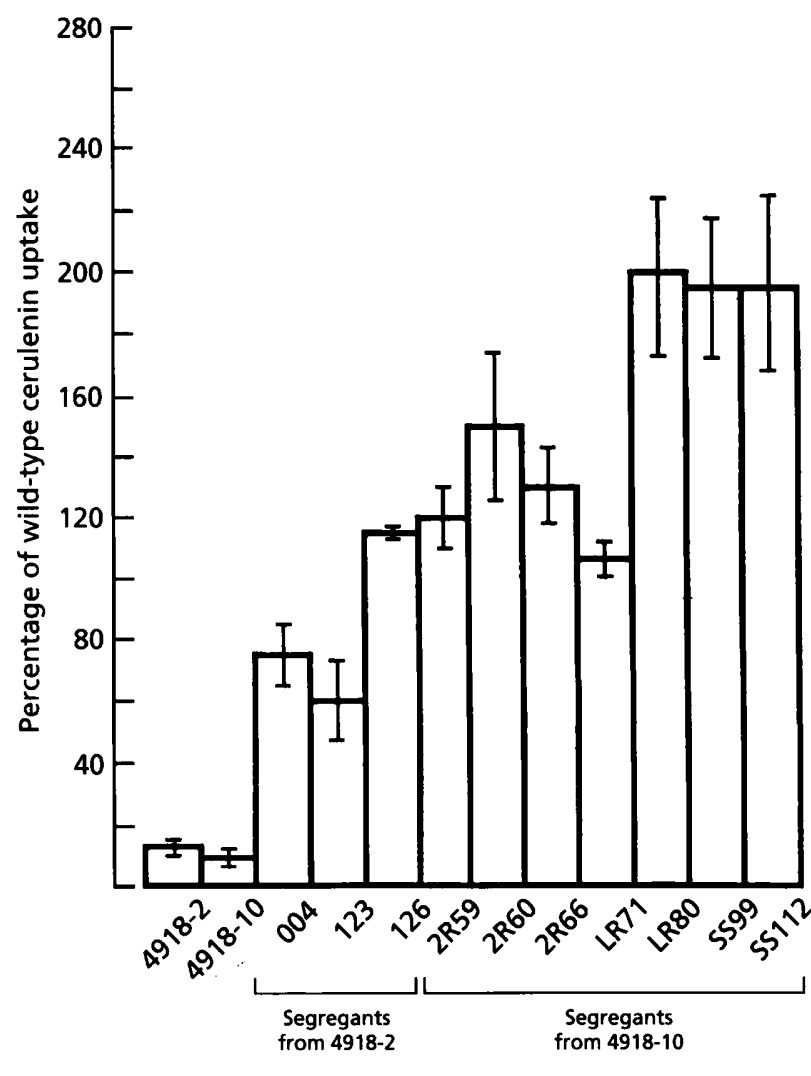

Fig. 3. Cerulenin uptake by cerulenin-sensitive segregants isolated from strains 4918-2 and 4918-10. The results presented are from three independent experiments, in which $5.0 \mathrm{ml}$ aliquots were collected $5 \mathrm{~min}$ after the introduction of $\left.{ }^{3} \mathrm{H}\right]$ cerulenin $\left(2.4 \mu \mathrm{Ci} \mu \mathrm{mol}^{-1} ; 88.8 \mathrm{kBq}^{-1} \mathrm{~mol}^{-1}\right)$. Uptake was then measured as described in Methods.

that cerulenin uptake by both strain 4918-2 and 4918-10 was approximately $24 \%$ of that of the parental strain. Similar experiments were performed utilizing ceruleninsensitive strains derived from 4918-2 and 4918-10. In all cases uptake by sensitive strains was significantly greater than that observed for strains 4918-2 and 4918-10 (Fig. 3).

\section{Cerulenin sensitivity of UV-induced segregants}

In order to ascertain if segregants acquired subsequent to UV-treatment were as sensitive to cerulenin as the parental 4918 strain, the MIC for cerulenin of the isolates was determined (Fig. 4). In all cases the MIC for the ceruleninsensitive strains was less than that exhibited by either strain 4918-2 or 4918-10; however, in nine out of ten strains tested, sensitivity to the wild-type level was not restored.

\section{DISCUSSION}

C. albicans strains $4918-2$ and $4918-10$ were isolated as spontaneous cerulenin-resistant mutants that were fortuitously avirulent in certain animal models of candidiasis (Calderone et al., 1986; Lehrer et al., 1986). While a variety of phenotypic differences between mutant and parental strains have been reported (Hoberg et al., 1986; Saxena $e t$ al., 1989; Malavasic et al., 1991), the basis of cerulenin resistance was not established. Cerulenin inhibits cell growth by irreversible binding to the condensing reaction domain of the $\alpha$ subunit of fatty-acid synthase (Ōmura, 1981 ; Funabashi et al., 1989). Thus, a possible mechanism to account for resistance is mutation to fatty-acid synthase such that enzyme affinity for cerulenin is reduced. Alternatively, changes previously reported in cell surface structure and composition (Hoberg et al., 1986; Saxena et al., 1989, 1990) could render the mutant strains less permeable to the agent.

Investigations utilizing parasexual techniques provided information concerning the genetic basis of cerulenin resistance. Results of UV-stimulated mitotic recombination experiments demonstrated that strain 4918-2 is heterozygous for cerulenin resistance $\left(\geqslant 5 \mu \mathrm{g} \mathrm{ml}^{-1}\right)$ and that resistance is dominant. In contrast, the change from resistance to sensitivity of strain $4918-10$ is reversible. Further analysis of strain 4918-10 in spheroplast fusion experiments did, however, demonstrate that cerulenin resistance was dominant. The former observation suggests that putative genetic changes in strain 4918-10 responsible for cerulenin resistance are not directed by simple allelic change from homozygosity to heterozygosity or vice versa. An alternative explanation is that reversibility might occur through a process analogous to that proposed to explain high-frequency switching of $C$. albicans colony morphology (Slutsky et al., 1985; Soll, 1992). It should be noted that strains $4918,4918-2$ anc 4918-10 all undergo colony morphology switching (Malavasic et al., 1991), but cerulenin resistance has not been correlated to a particular colony type (R. L. Cihlar, unpublished).

The data indicate that changes to fatty-acid synthase and in permeability may both contribute to cerulenin resistance of strains 4918-2 and 4918-10. In the latter instance, the uptake of cerulenin by the mutant strains was approximately $24 \%$ of that of strain 4918 . In addition, cerulenin uptake was restored to varying degrees (e.g. 0.63-2.0-times wild-type levels) in UV-stimulated cerulenin-sensitive segregants. Thus the data support the contention that reduction in cerulenin uptake contributes significantly to cerulenin resistance of strains 4918-2 and 4918-10, although it remains possible that UV-treatment may have resulted in new mutations affecting permeability.

Evidence was reported previously that suggested cerulenin resistance of strains 4918-2 and 4918-10 may, in part, be attributable to mutation of fatty-acid synthase (McElhaney-Feser \& Cihlar, 1994). This proposal emanated from inhibition studies demonstrating differences in cerulenin-mediated inactivation of fattyacid synthase from the mutant strains when compared to the enzyme from the parental strain. In the present study inactivation kinetics of fatty-acid synthase from UVstimulated cerulenin-sensitive segregants showed no significant variance from those of 4918-2 and 4918-10. Thus, strain sensitivity to cerulenin was restored despite 


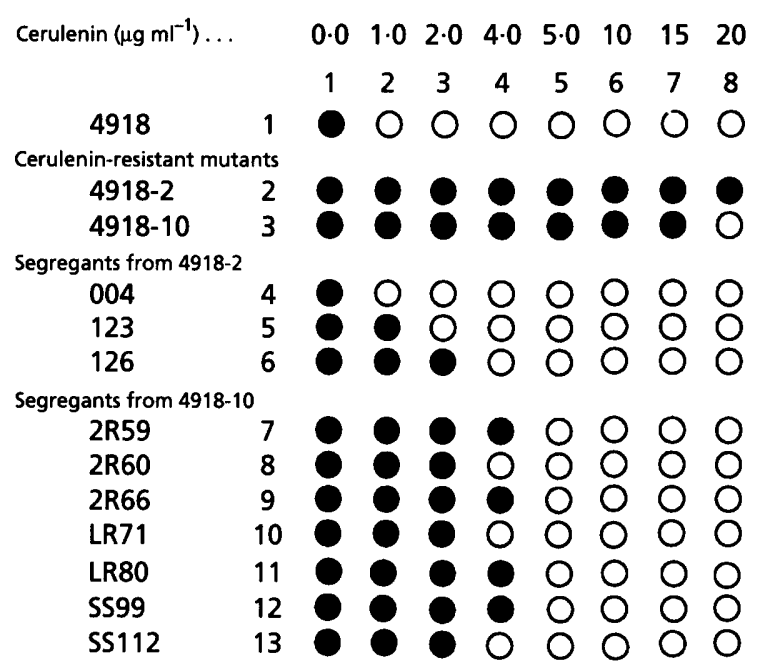

Fig. 4. Determination of the MIC for cerulenin for strains 4918, 4918-2, 4918-10 and UV-stimulated cerulenin-sensitive segregants. Growth; $O$, no growth.

apparent resistance of fatty-acid synthase to cerulenin. A possible explanation for this paradox can be reasoned from the results of cerulenin MIC determinations for each cerulenin-sensitive segregant. In particular, segregants were identified by failure to grow on minimal media supplemented with $5 \mu \mathrm{g}$ cerulenin $\mathrm{ml}^{-1}$, the condition used initially in isolation of 4918-2 and 4918-10 (Cihlar et al., 1984). However, the MIC of nine out of ten segregants was greater than that of strain 4918 , but less than the $5 \mu \mathrm{g} \mathrm{ml}^{-1}$ used in selections. This result suggests that alteration of fatty-acid synthase in strains 4918-2 and 4918-10 maintains resistance to low concentrations of cerulenin (e.g. less than $5 \mu \mathrm{g} \mathrm{ml}^{-1}$ ), while changes in permeability offer resistance to substantially higher concentrations of the agent. This would also imply that two independent genetic changes are responsible for the resistance levels observed. In this regard, Inokoshi et al. (1994) showed that cerulenin resistance of a Saccharomyces cerevisiae strain was due to a missense mutation in FAS2 that resulted in an amino acid substitution 55 amino acids upstream from the cerulenin-binding site of fatty-acid synthase. No mutations have been detected in the same region of C. albicans F AS2 of either strain 4918-2 or 491810 (Southard \& Cihlar, 1995), but it is likely that mutation at other positions could result in increased cerulenin resistance of the enzyme. These issues, in addition to the possible relationship of other phenotypic changes to specific genetic alterations, remain under investigation.

\section{ACKNOWLEDGEMENTS}

This work was supported by Public Health Service Grants DE00189 and PO1 AI37251 from the National Institutes of Health.

\section{REFERENCES}

Calderone, R. A., Cihlar, R. L., Lee, D. D. S., Hoberg, K. \& Scheld, M. (1986). Yeast adhesion in the pathogenesis of endocarditis due to Candida albicans: studies with adherence-negative mutants. $J$ Infect Dis 152, 710-715.

Cihlar, R. L., Hoberg, K. \& Calderone, R. A. (1984). Isolation of cerulenin-resistant mutants of Candida albicans. In Microbiology-1984, pp. 148-149. Edited by L. Levine \& D. Schlesinger. Washington, DC: American Society for Microbiology.

Cutler, J. E. (1991). Putative virulence factors of Candida albicans. Annu Rev Biochem 45, 187-218.

De Bernardis, F., Adriani, D., Lorenzini, R., Pontieu, E., Carruba, G. \& Cassone, A. (1993). Filamentous growth and elevated vaginopathic potential of a nongerminative variant of Candida albicans expressing low virulence in systemic infection. Infect Immun 61, 1500-1508.

Funabashi, H., Kawaguchi, A., Tomoda, H., Ōmura, S., Okuda, S. \& Iwasaki, S. (1989). Binding site of cerulenin in fatty acid synthase. $J$ Biochem 105, 751-755.

Goshorn, A. K. \& Scherer, S. (1989). Genetic analysis of prototrophic natural variants of Candida albicans. Genetics 123, 667-673.

Hoberg, K. A., Cihlar, R. L. \& Calderone, R. A. (1986). Characterization of cerulenin-resistant mutants of Candida albicans. Infect Immun 51, 102-109.

Hostetter, M. K. (1994). Adhesions and ligands involved in the interaction of Candida spp. with epithelial and endothelial surfaces. Clin Microbiol Rev 7, 29-42.

Inokoshi, J., Tomoda, H., Hashimoto, H., Watanabe, A., Takeshima, H. \& Omura, S. (1994). Cerulenin-resistant mutants of Saccharomyces cerevisiae with an altered fatty acid synthase gene. Mol \& Gen Genet 244, 90-96.

Lehrer, N., Segal, E., Cihlar, R. L. \& Calderone, R. A. (1986). Pathogenesis of vaginal candidiasis: a mutant which has reduced ability to adhere in vitro. J Med Vet Mycol 24, 127-131.

Lynen, F. (1969). Yeast fatty acid synthase. Methods Enzymol 14, 17-33.

Malavasic, M. J., McElhaney-Feser, G. E., Southard, S. B. \& Cihlar, R. L. (1991). Phenotypic variation of a virulent Candida albicans strain and two spontaneous, relatively avirulent mutant strain derivatives. J Med Vet Mycol 29, 145-155.

Manning, M. \& Mitchell, T. G. (1980). Strain variation and morphogenesis of yeast and mycelial-phase Candida albicans in low sulfate, synthetic medium. J Bacteriol 142, 714-719.

McElhaney-Feser, G. E. \& Cihlar, R. L. (1994). Purification and characterization of fatty acid synthase from Candida albicans, strain 4918, and two derived spontaneous cerulenin-resistant mutants. $J$ Med Vet Mycol 32, 13-20.

Miller, J. H. (1972). Experiments in Molecular Genetics. Cold Spring Harbor, NY: Cold Spring Harbor Laboratory.

Odds, F. C. (1988). Candida and Candidosis. London: Baillière Tindall.

Ollert, M. W., Wadsworth, E. \& Calderone, R. A. (1990). Reduced expression of the functionally active complement receptor for iC $3 \mathrm{~b}$ on an avirulent mutant of Candida albicans. Infect Immun 58, 909-913.

Omura, S. (1981). Cerulenin. Methods Enzymol 72, 520-532.

Ross, I. K., De Bernardis, F., Emerson, G. W., Cassone, A. \& Sullivan, P. A. (1990). The secreted aspartate proteinase of Candida albicans: physiology of secretion and virulence of a proteinase deficient mutant. J Gen Microbiol 136, 687-694.

Saxena, A., Hammer, C. F. \& Cihlar, R. L. (1989). Analysis of mannans of two relatively avirulent strains of Candida albicans. Infect Immun 57, 413-419. 
Saxena, A., McElhaney-Feser, G. E. \& Cihlar, R. L. (1990). Mannan composition of the hyphal form of two relatively avirulent mutants of Candida albicans. Infect Immun 58, 2061-2066.

Slutsky, B., Buffo, J. \& Soll, D. R. (1985). High-frequency switching of colony morphology in Candida albicans. Science 230, 666-669.

Soll, D. R. (1992). High frequency switching in Candida albicans. Clin Microbiol Rev 5, 183-203.
Southard, S. B. \& Cihlar, R. L. (1995). Analysis and expression of the Candida albicans F AS2 gene. Gene 156, 133-138.

Whelan, W. L. \& Magee, P. T. (1981). Natural heterozygosity in Candida albicans. J Bacteriol 145, 896-903.

Received 14 October 1994; revised 3 January 1995; accepted 6 April 1995. 